\title{
Covalent and non-covalent strategies for the immobilization of Tobacco Etch Virus (TEV) protease on superparamagnetic nanoparticles
}

Jessica L. Norris, Tulsi Patel, Anvesh K. R. Dasari, Thomas A. Cope, Kwang Hun Lim, Robert M. Hughes*. Department of Chemistry, East Carolina University, Greenville, North Carolina, United States 27858-4353 *hughesr16@ecu.edu

Proteases with highly specific activities have numerous applications, including the cleavage of affinity tags (Flag; HA; His6X) and solubility promoting partners (GST; MBP) within the context of protein isolation and purification schemes. However, commercially sourced proteases such as Tobacco Etch Virus (TEV) protease and Human Rhinovirus (HRV) 3C protease are typically applied as single use aliquots, which limits their cost-effectiveness. In addition, the presence of residual proteases can complicate downstream applications and analyses of the protein(s) of interest. Thus, the creation of immobilized, reusable site-specific proteases would be of significant value to the life science community. In this work, we use two strategies for the immobilization of TEV protease onto superparamagnetic iron oxide nanoparticles (SPIONs). In one strategy, a MBP-TEVStreptavidin fusion protein is immobilized on biotin-functionalized SPIONs. In second strategy, TEV protease is coupled onto SPIONs non-directionally, via amine-mediated attachment, and directionally, via HALO-tag mediated attachment. We demonstrate activity of our immobilized proteases in the presence of a MBP-GFP fusion protein containing the TEV protease target sequence (ENLYFQ|S). We then analyzed time-dependent activity, longevity, and reuse of these immobilized protein preparations, comparing and contrasting each approach. The protease immobilization strategies described in this work may be useful tools for simplifying challenging protein purification protocols, in addition to providing general methods for enzyme immobilization on SPIONs.

\section{INTRODUCTION}

Tobacco Etch Virus protease (TEVp) is a site specific protease that cleaves proteins containing the amino acid sequence ENLYFQ|S/G.[1] Early on, undesirable properties of the native protease limited its potential applications in biochemistry and molecular biology. For example, native TEVp undergoes autolysis, rapidly rendering the protease inactive. In addition, as a cysteine protease, TEVp is highly sensitive to oxidation. To address this, variants of TEVp have been generated that greatly reduce autolysis (e.g. TEV mutants S219V \& S219P), while activity loss due to cysteine oxidation can be easily suppressed via reductants such as DTT or $\beta$-ME.[2] As a result of these and other efforts[3, 4], engineered TEVp variants are now valuable tools for the site specific removal of solubility and affinity tags in protein expression and purification schemes, in addition to having applications in the design and development of biochemical switches for the control of cell signaling pathways $[5,6]$. Commercially available TEVp preparations typically contain an affinity tag (6XHis; GST), enabling capture of the protease, post-cleavage of the target protein. However, without the incorporation of additional steps, protein purification strategies utilizing these affinity labeled TEV proteases may still suffer from the presence of residual TEVp post purification[7]. An immobilized TEVp would not only eliminate downstream protease contamination, but also simplify protein preparations by eliminating the need to remove TEVp via column chromatography. However, 
despite the potential advantages of TEVp immobilization, there are currently no commercially available preparations of immobilized TEVp. Previously reported immobilization strategies for TEVp include the creation of a TEV-Streptag fusion and subsequent immobilization on StrepTactin-coated agarose beads[8], and a unique strategy for the deposition of TEVp onto amyloid fiber coated glass beads[9]. While both of these strategies resulted in immobilized TEV with retention of activity and specificity, both exhibit loss of activity with repeated use, illustrating a key challenge encountered by many enzyme immobilization strategies. In order to better understand this challenge, and in an effort to create alternative solutions for TEV immobilization, we explored two different strategies for the TEVp immobilization onto superparamagnetic iron oxide nanoparticles (SPIONs), an attractive substrate for enzyme immobilization due to their high surface area, ease of recovery, and potential for biomedical applications[10, 11]. In one strategy, we assemble TEVp-streptavidin (TEV-STRPT) and TEVp-TRPTavidin (TEV-TRPT) fusion proteins, and explore their proteolytic activity when non-covalently immobilized onto biotinylated SPIONs. In a second strategy, we utilize standard NHS ester bioconjugation chemistry to covalently attach TEVp onto SPIONs. In a third strategy, we utilized a TEV-HALOtag fusion to covalently attach TEVp onto SPIONs. By comparing and contrasting non-covalent immobilization strategies with covalent strategies, we identify the challenges associated with each strategy while further developing methods for TEVp immobilization.

\section{MATERIALS AND METHODS}

PCR AND GENE CLONING Gene fragments (MBP-TEV and Streptavidin, TRPTavidin, or HALOtag) and target expression vector (pNic28) were amplified via polymerase chain reaction using Q5 polymerase (NEB) and subsequently gel isolated. Gene fragment and expression vector amplicons were then combined in a 2:2:1 ratio ( $4 \mu \mathrm{L}$ MPB-TEV; $4 \mu \mathrm{L}$ Streptavidin; $2 \mu \mathrm{L}$ pNic28), followed by the addition of $10 \mu \mathrm{L}$ of 'Hot PFX' buffer (a modification of the Hot Fusion cloning method [12]: $200 \mu \mathrm{L}$ of $0.5 \mathrm{M}$ Tris HCl, pH 7.4; $200 \mu \mathrm{L}$ of $50 \mathrm{mM} \mathrm{MgCl}$; $20 \mu \mathrm{L}$ dNTPs (10 mM stock); $10 \mu \mathrm{L}$ DTT (1M stock); 50 mg PEG 4000; $2 \mu \mathrm{L}$ of Accuprime PFX added per $100 \mathrm{uL}$ of $2 X$ Buffer) to give a 20 $\mu \mathrm{L}$ solution in a thin-walled PCR tube. The solution was incubated for $1 \mathrm{~h}$ at $50^{\circ} \mathrm{C}$ in a PCR thermal cycler (Eppendorf) and then cooled to $4{ }^{\circ} \mathrm{C}$ prior to transformation. Transformation into ultracompetent cells (Top 10 Gold (Stratagene)) followed, conducted according to manufacturer's protocols. The resulting transformation mixture was plated in its entirety on LB agar (Kanamycin) plates and incubated overnight at $37{ }^{\circ} \mathrm{C}$. Resultant colonies were then grown overnight in $4 \mathrm{~mL}$ liquid culture (Terrific Broth (Kanamycin)) and plasmid DNA was isolated by standard spin column purification. Plasmids were characterized by restriction digest (Hind III/Ndel (NEB)) for correct insert size, followed by Sanger sequencing.

SOURCE PLASMIDS Plasmid templates for gene construction were obtained from Addgene, Inc., a non-profit DNA repository: pRK793 was a gift from David Waugh (Addgene plasmid \# 8827; http://n2t.net/addgene:8827 ; RRID:Addgene_8827)[2]; pET MBP GFP LIC cloning vector (MBPmsfGFP) was a gift from Scott Gradia (Addgene plasmid \# 29750; http://n2t.net/addgene:29750; RRID:Addgene_29750); pET21a-TRPTavidin was a gift from Mark Howarth (Addgene plasmid \# 
24753 ; http://n2t.net/addgene:24753; RRID:Addgene_24753)[13]; pENTR4-HaloTag (w876-1) was a gift from Eric Campeau (Addgene plasmid \# 29644 ; http://n2t.net/addgene:29644 ; RRID:Addgene_29644)

PROTEIN EXPRESSION AND PURIFICATION Sequence verified plasmids were transformed into BL$21 \mathrm{DE} 3 \mathrm{E}$. coli for protein expression. $10 \mathrm{~mL}$ starter cultures, grown overnight at $30^{\circ} \mathrm{C}$, were used to inoculate Terrific Broth - Kanamycin cultures $(500 \mathrm{~mL})$. Cultures were grown at $37^{\circ} \mathrm{C}$ with shaking to $\mathrm{OD}_{600} \sim 1.2$, and then cooled to $25^{\circ} \mathrm{C}$. Protein expression was induced with $1 \mathrm{mM}$ IPTG, followed by $20 \mathrm{~h}$ of shaking $(260 \mathrm{rpm})$. Resulting bacterial cultures were pelleted by centrifugation (20 min; $5000 \mathrm{rpm} ; 4^{\circ} \mathrm{C}$ ), and then lysed by $10 \mathrm{~mL}$ of B-PER with added protease inhibitors. Lysed cultures were centrifuged (20 min; $14000 \mathrm{rpm} ; 4^{\circ} \mathrm{C}$ ), and the resulting supernatant and pellets separated. For the MBP-GFP substrate protein and the MBP-TEVS219V protein, the resulting lysates were purified via IMAC chromatography (Cobalt resin), followed by size exclusion chromatography. For the TEVp-STRPT and TEVp-TRPT proteins, the pellet fraction was subjected to an inclusion body prep, while the clarified lysate fraction was used without further purification. Briefly, the inclusion body prep was conducted as follows: the bacterial pellet was resuspended in B-PER (10 mL). Lysozyme ( $200 \mu \mathrm{L}$ of a $10 \mathrm{mg} / \mathrm{mL}$ stock; $50 \%$ glycerol solution) was added to the solution, which was then mixed well and incubated for $5 \mathrm{~min}$ at room temperature. The solution was then diluted with $50 \mathrm{~mL}$ of diluted B-PER reagent (diluted 1:10) and mixed by vortexing. The inclusion bodies were harvested by centrifugation ( $15 \mathrm{~min} ; 14000 \mathrm{rpm} ; 4^{\circ} \mathrm{C}$ ). The resulting inclusion body pellets were then resuspended in $50 \mathrm{~mL}$ 1:10 diluted B-PER reagent. Solutions were then sonicated on ice to reduce viscoscity/pellet clumping. This resuspension and centrifugation step was repeated two more times. The resulting inclusion bodies were then solubilized in $5 \mathrm{~mL}$ of HEPES solubilization buffer (50 mM Hepes ( $\mathrm{pH} \mathrm{7.5);6} \mathrm{M} \mathrm{Guanidinium} \mathrm{HCl;25} \mathrm{mM} \mathrm{DTT)} \mathrm{and}$ incubated on ice for $1 \mathrm{~h}$, followed by centrifugation (14000 rpm; $10 \mathrm{~min} ; 4^{\circ} \mathrm{C}$ ) to remove any remaining insoluble material. The stock protein solution (concentration determined by Bradford assay) was diluted to $1 \mathrm{mg} / \mathrm{mL}$ in the HEPES solublization buffer, followed by dropwise dilution into refolding buffer ( $5 \mathrm{~mL}$ sample into $45 \mathrm{~mL}$ refolding buffer; $50 \mathrm{mM}$ glycine $(\mathrm{pH}$ 9)/200 mM $\mathrm{NaCl} / 5 \mathrm{mM}$ EDTA/10 mM DTT/1X protease inhibitors) on ice. The resulting solution was mixed vigorously for $2 \mathrm{~min}$, insoluble material removed by centrifugation, and the remaining soluble sample further concentrated by spin concentration. MBP-TEV-HALO expression: $500 \mathrm{~mL}$ cultures of MagicMedia (Invitrogen) were prepared according to manufacturer's instructions using sterile technique. $5 \mathrm{~mL}$ starter cultures in LB media were used for inoculation. The culture was grown to $\mathrm{OD}_{600}=2.5\left(300 \mathrm{rpm}, 37^{\circ} \mathrm{C}\right)$, then the incubator temperature was lowered to $25^{\circ} \mathrm{C}$. Autoinduction was allowed to continue for 29 hours $\left(300 \mathrm{rpm}, 25^{\circ} \mathrm{C}\right.$ ), followed by harvest and lysis as described above.

PROTEIN CHARACTERIZATION Purity of protein isolated from the inclusion body prep was characterized via SDS-PAGE. The band corresponding to the TEV-STRP fusion protein was excised and digested with trypsin overnight using a standard in-gel digestion protocol. The resultant tryptic peptides were desalted using a C18 Zip-Tip and applied to a MALDI target plate with $\alpha$-cyano-4hydroxycinnamic acid as the matrix. The sample was analyzed on an AB Sciex 5800 MALDI TOF/TOF 
mass spectrometer. MS/MS spectra were searched against Uniprot databases using Mascot version 2.3 (Matrix Science) within ProteinPilot software version 3.0 (AB Sciex). A significance score threshold was calculated in Mascot, with ion scores above the threshold considered positive IDs $(p<0.05)$. Peptides corresponding to both TEVp and Streptavidin were identified in the analysis (Supporting Table 1). To confirm TEV protease activity, the TEV-SA fusion protein was then assayed using a fluorescent assay of TEV protease activity (Sensolyte ${ }^{\circledR}$ TEV Protease Assay Kit). Briefly, $50 \mu \mathrm{L}$ of refolded, spin concentrated TEV-STRPT protein $(0.5 \mathrm{mg} / \mathrm{mL})$ was combined with $80 \mu \mathrm{L}$ of TEV assay buffer, including pro-fluorescent TEV substrate (5-FAM/QXL 520) and DTT, following manufacturer's instructions. Fluorescence was monitored (Ex/Em $=490 \mathrm{~nm} / 520 \mathrm{~nm}$ ) every 30 s with a Molecular Devices M2 plate reader.

NANOPARTICLE LOADING AND CHARACTERIZATION TEV-STRPT-Biotin-SPIONs and TEV-TRPTBiotin-SPIONs: Biotin-labeled superparamagnetic nanoparticles $(500 \mu \mathrm{L}$ of a $5 \mathrm{mg} / \mathrm{mL}$ solution (RayBiotech)) were pelleted on a magnetic separator, storage solution removed by pipette, and washed with PBS $(3 \times 1 \mathrm{~mL})$. After removal of the last PBS wash, pelleted beads were resuspended in $1 \mathrm{~mL}$ of PBS and transferred to a $15 \mathrm{~mL}$ conical tube. Clarified bacterial lysates $(8 \mathrm{~mL}$ of a 3 $\mathrm{mg} / \mathrm{mL}$ solution in M-PER) were added to the beads, which were incubated at room temperature on an end-over-end rotor for $2.5 \mathrm{~h}$. Beads were pelleted on a magnetic separator, lysate removed, and beads washed with PBS ( $3 \times 5 \mathrm{~mL} ; 10 \mathrm{~min}$ each). After the last wash, pelleted beads were resuspended in $1 \mathrm{~mL}$ PBS and transferred to a $1.5 \mathrm{~mL}$ centrifuge tube. Beads were pelleted again, followed by removal of PBS and resuspension of beads in $500 \mu \mathrm{L}$ of TEV storage buffer. Beads were stored at $4{ }^{\circ} \mathrm{C}$ for later use. By eluting bound TEVp-STRPT from loaded SPIONs, fusion protein loading was determined to be $0.003(+/-0.001) \mathrm{mg}$ protein/mg SPION, approximately $1 / 10^{\text {th }}$ of the maximum SPION binding capacity. TEVS219V-SPIONs: NHS activated superparamagnetic beads (7.5 mg of a $50 \mathrm{mg}$ lyophilized powder containing $2.5 \mathrm{mg}$ beads; OceanNanotech) were resuspended in $1 \mathrm{~mL}$ of buffer (PBS with $0.1 \%$ Tween-20) in a $15 \mathrm{~mL}$ conical tube. Prepared TEV S219V (2 mL of a $0.1 \mathrm{mg} / \mathrm{mL}$ solution in PBS, pH 7.4) was added to the bead solution and incubated at room temperature on an end-over-end rotator for $1 \mathrm{~h}$. Beads were pelleted on a magnetic separator and the incubation solution removed by pipette. The beads were then washed $(3 \times 5$ $\mathrm{mL}$; PBS with $0.1 \%$ Tween-20), followed by resuspension in TEV storage buffer (200 $\mu \mathrm{L}$ final volume; $1.875 \mathrm{mg} / \mathrm{mL}$ ). TEV-HALO-SPIONs: Chloroalkane functionalized nanoparticles (200 $\mu \mathrm{L}$ of a $20 \%$ slurry of macroporous cellulose encapsulated SPIONs (Promega)) were pelleted on a magnetic separator, storage solution removed by pipette, and washed with PBS $(3 \times 1 \mathrm{~mL})$. After removal of the last PBS wash, pelleted beads were resuspended in $1 \mathrm{~mL}$ of PBS and transferred to a $15 \mathrm{~mL}$ conical tube. Clarified bacterial lysates ( $5 \mathrm{~mL}$ of a $3 \mathrm{mg} / \mathrm{mL}$ solution in M-PER) were added to the beads, which were incubated at room temperature on an end-over-end rotor for $2.5 \mathrm{~h}$. Beads were pelleted on a magnetic separator, lysate removed, and beads washed with PBS ( $3 \times 5$ $\mathrm{mL} ; 10$ min each). After the last wash, pelleted beads were resuspended in $1 \mathrm{~mL}$ PBS and transferred to a $1.5 \mathrm{~mL}$ centrifuge tube. Beads were pelleted again, followed by removal of PBS and resuspension of beads in $200 \mu \mathrm{L}$ of TEV storage buffer. Beads were stored at $4{ }^{\circ} \mathrm{C}$ for later use. TEM sample preparation and sample acquisition TEM grids were prepared by loading $10 \mu \mathrm{L}$ droplets 
of SPIONs in $\mathrm{ddH}_{2} \mathrm{O}$ on formvar/carbon coated copper 400 mesh grids (Electron Microscopy Sciences, Inc.). Droplets (on grids) were then frozen on stainless steel cylinders surrounded by a liquid $\mathrm{N}_{2}$ bath, followed by freeze drying in a Labconco lyophilizer. The grids were then imaged under Philips CM12 Transmission Electron Microscope with an accelerated voltage of $80 \mathrm{kV}$.

IMMOBILIZED ENZYME ASSAYS Nanoparticle preparations $(5 \mathrm{mg} / \mathrm{mL}$ solution, suspended in TEV storage buffer (20 mM Tris-HCl, pH 8; 300 mM NaCl; 1 mM EDTA; 50\% glycerol; 10 mM DTT or 5 $\mathrm{mM} \beta M E)$, were combined with MBP-ENLYFQS-GFP substrate $(0.1-0.2 \mathrm{mg} / \mathrm{mL}$ in PBS). DTT or $\beta M E$ was added to a final concentration of $10 \mathrm{mM}$ (DTT) or $5 \mathrm{mM}(\beta M E)$. Assays were incubated in a $30^{\circ} \mathrm{C}$ incubator on an end-over-end rotator. To harvest cleaved substrate protein, beads were pelleted on a magnetic separator (ThermoFisher MagJet), and supernatants removed by pipette. For bead re-use assays, pelleted beads were resuspended in $20 \mu \mathrm{L}$ of TEV storage buffer prior to the addition of MBP-GFP substrate and reductant (10 mM DTT or $5 \mathrm{mM} \mathrm{\beta ME).}$

PROTEIN ELECTROPHORESIS AND WESTERN BLOTTING SDS-PAGE Protein separations were conducted on 10\% Acrylamide Gels (made via standard protocol) or 4-12\% gradient gels (Expedeon, Inc.) as indicated. Gels were washed 3X with ddH2O prior to staining with Safestain (Invitrogen). Western blot transfers onto PVDF membranes were conducted overnight at 20V. Membranes were blocked with 5\% BSA/TBS-T prior to incubation with primary antibody (1: 1000 diultion, Santa Cruz Biotechnologies Sc-8334; $4{ }^{\circ} \mathrm{C}$ overnight). Blots were washed with TBST ( $3 \times 5$ $\min$ ) prior to incubation with secondary antibody. After a final wash (3X TBS-T), blots were incubated with chemiluminescent substrate [14] and imaged with an Azure cSeries imager.

\section{RESULTS AND DISCUSSION}

To create an immobilized TEV protease (Figure 1A), we cloned a protein expression cassette consisting of MBP, TEV protease S219V, and streptavidin (Figure 2A) into the bacterial expression vector pNic28, by fusing the ORF from a previously validated MBP-TEV construct [2] to that of a synthetic streptavidin gene. The MBP tag is necessary for promoting soluble TEV production, and is separated from the TEV protease via a TEV cleavage site, promoting in situ cleavage of the solubility tag. Following established protocols for TEV protease expression [15], we investigated whether the same protein expression and isolation protocols could produce an active TEVp-STRPT fusion protein. After protein expression and lysis of the resulting bacterial pellet, an inclusion body preparation method was used to recover and refold insoluble TEV-STRP (Fig. 2B; (apparent MW $\sim 45$ kD; calculated MW: 45221.61 Daltons)). The resulting protein was assayed for TEV activity using a pro-fluorescent TEV substrate and found to possess TEV protease activity (Fig. 2C). Concurrently, a gel band containing the same protein was excised and characterized by tryptic digest and MS analysis of the resulting peptide fragments (Fig. 2D and Supporting Table 1). This study identified peptides corresponding to both TEVp and STRP, indicating successful expression and isolation of the TEVp-STRPT protein fusion.

Following confirmation of TEVp-STRPT fusion expression and activity, we investigated the ability of the STRPT tag to serve as an immobilizing anchor on solid support. Using commercially available 
biotin-labelled superparamagnetic iron oxide nanoparticles (SPIONs), soluble TEVp-STRPT fusion protein was isolated directly from bacterial lysates [16]. Following thorough washing, the resulting SPIONs were then assayed for TEVp activity using an MBP-GFP protein fusion with a TEV recognition site between the MBP and GFP domains. Cleavage of the MBP-GFP fusion in the presence of TEVp-STRPT-biotin SPIONs was assessed with anti-GFP western blot and compared to control solutions without TEVp-STRPT-biotin SPIONs (Fig. 3A-B). As an additional control, beads loaded with a non-protein overexpressing bacterial lysate were prepared using an identical loading and washing method. In comparison to the TEVp-STRPT-biotin SPIONS, these SPIONs were found to have no background TEVp activity (Fig. 3C).

Having confirmed the activity of TEVp when immobilized on SPIONs via streptavidin-biotin conjugation, we then sought to test the longevity of the TEVp SPION preparation. After $>1$ month in cold storage $\left(4^{\circ} \mathrm{C}\right)$, we observed no loss of TEVp activity (Fig. $\left.4 \mathrm{~A}\right)$. We note that addition of reductant (10 $\mathrm{mM}$ DTT or $5 \mathrm{mM} \mathrm{BME}$ ) to each assay was required to restore TEV activity after several days in cold storage. However, these promising results were tempered by the presence of an unexpected band on a 10\% resolving gel, appearing near the MBP cleavage fragment (Fig. 4B). We suspected that this band was due to loss of the TEV-STRPT fusion protein from biotinylated SPIONs, as its apparent MW corresponded to the previously characterized electrophoretic mobility of the TEVp-STRPT fusion isolated from inclusion bodies. As an additional control experiment, TEVP-STRPT-biotin SPIONs were incubated alone in solution (without MBP-GFP substrate), and the resulting supernatant analyzed by SDS-PAGE. The unexpected band was also present in these control solutions, thus confirming leakage of TEVp-STRPT from the SPIONs. Loss of TEVp-STRPT from beads was further supported by reuse assays of TEVp SPIONS. In these assays, the SPIONs lost significant activity over three successive trials (Trial 1: 100\% activity; Trial 2: 40\% of initial activity; Trial 3: 15\% of initial activity; Supporting Figure 1), consistent with loss of TEVp fusion protein during each successive trial. This result is also consistent with literature data for dissociation of the streptavidin-biotin complex over extended time intervals, in which 50\% of the streptavidin-biotin complex dissociates $48 \mathrm{~h}$ post-formation [17].

In order to circumvent this problem, two strategies were proposed for reduction or elimination of TEVp-STRPT loss. The first used formaldehyde as a fixative for the SPIONs, following TEVP-STRPT loading and washing. This strategy borrows from early methods of enzyme mobilization, in which aldehyde fixatives were used to sequester proteins onto solid supports, with surprising retention of activity in many cases[18, 19]. Initially, it appeared that this strategy was successful, as increasing fixation times resulted in SPIONs that retained TEV activity, yet exhibited less loss of the TEVp-STRPT fusion when compared to unfixed SPION preparations (Supporting Figure 2A-B). However, after 10 days in cold storage, these PFA-fixed SPIONs exhibited significantly more loss of the TEVP-STRPT fusion when comparted to non-fixed SPIONs, as demonstrated by assay of the SPION storage buffer alone (Supporting Figure $2 \mathrm{C}$ ). Thus, the fixation strategy was abandoned as a viable approach to ensuring long term SPION stability. 
The second strategy to create a more stable TEVp SPION was to incorporate Traptavidin (TRPT) into the TEVp protein fusion in place of STRPT (Fig. 1B). Traptavidin is a recently reported mutant of streptavidin with a lower $k_{\text {off }}$ rate for biotin binding versus streptavidin [13]. These mutations effectively increase the overall residence time of the complex with biotin, making Traptavidin a potentially more attractive alternative to streptavidin for biomaterials applications [20]. To test this, we constructed an expression cassette for an MPB-TEVS219V-TRPT fusion protein, expressing and immobilizing this protein fusion as previously described. However, side-by-side assays of the resulting TEVp-TRPT SPIONs with the TEVp-STRPT SPIONs demonstrated that TEVp-TRPT SPIONs were unable to cleave the MBP-GFP substrate protein with the same efficiency as the TEVP-STRPT SPIONs (Supporting Figure 2D). We were unable to improve upon this result despite numerous efforts to increase the soluble expression and loading of the TEVp-TRPT protein fusion. Furthermore, SPIONs bearing the TEVp-TRPT fusion suffered from a similar loss of activity upon reuse as their STRPT counterparts (Fig. 5), indicating that TEVp fusion loss was not completely eliminated with the TEVp-TRPT module.

Having identified deficiencies in our non-covalent approach to TEVp immobilization, we then turned to covalent methods for TEVp capture onto SPIONs. Two potential strategies were identified: non-directional covalent coupling of TEVp onto SPIONs by coupling of primary amines to NHS-ester functionalized SPIONs, and directional covalent coupling of TEVp onto SPIONS via protein-alkyl linkage via a TEV-HALOtag fusion protein. To execute the first strategy, purified TEVp S219V was incubated with NHS ester functionalized nanoparticles, followed by extensive washing. We anticipated that this strategy might provide a simple, direct solution to TEVp immobilization that circumvented the issues associated with expression of a TEVp fusion protein. However, in side-by-side comparisons of SPIONs generated with the covalent, non-directional coupling strategy with SPIONs generated using the non-covalent, directional coupling strategy, direct TEVp coupling failed to match the STRPT and TRPT beads in both substrate cleavage efficiency and reusability (Fig. 5).

The second covalent strategy was executed by cloning an expression cassette for a MPB-TEVpHALOtag fusion. In this strategy, we anticipated that the HALOtag would provide a highly selective means of protein fusion immobilization, while eliminating the solubility issues posed by STRPT and TRPT[21]. In addition, prior literature demonstrates that enzymes generally tolerate HALOtag fusion with good retention of activity[16, 22]. Following protein expression, the TEVp-HALO fusion was captured from bacterial lysate using commercially available chloroalkane functionalized SPIONs, followed by extensive washing. This approach resulted in SPIONs with high cleavage efficiency (Fig. 6A) and significantly prolonged reusability (Fig. 6B). Furthermore, in constrast to TEVP-STRPT and TEVp-TRPT SPIONs, no fusion protein loss was detected on SDS-PAGE gels (Fig. 6A). In extended reuse assays, the TEVp-HALO-SPIONs maintained $100 \%$ activity over four successive trials (Fig. 6B), with significant TEVp activity loss occurring only after six successive trials (Fig. 7). TEM images of the biotinylated and NHS ester-functionalized SPIONs both before and after protein immobilization demonstrate that biotinylated and direct coupled SPIONs undergo significant aggregation and morphological change as a result of protein loading (Supporting Figure 
3). By contrast, the macroporous cellulose encapsulated SPIONs used for HALO capture are well dispersed and undergo no detectable changes in size (Supp. Fig 3). Therefore, while the superior performance of the TEVp-HALO-SPIONs is primarily due to directional covalent capture of the TEVp-HALO fusion, inhibition of SPION aggregation due to cellulose encapsulation may also play a role in their enhanced properties.

In this work, we have investigated several methods for the immobilization of TEVp onto SPIONs. Based on the criteria of reusability and fusion protein retention, the HALO tagging strategy was most promising, yielding an easily recoverable reagent that maintains enzymatic activity over numerous applications. In addition, it is likely that this strategy is amenable to large scale production, making it a potentially viable reagent for routine use in biochemistry labs. However, despite the shortcomings of the other approaches investigated, their potential utility should not be ignored. The STRPT and TRPT protein fusions, for example, may have applications for in vivo delivery of enzyme labelled SPIONs, in which slow release of the enzyme at the target site is desired [23]. Over time, TEV protease has acquired a reputation of being rather difficult to immobilize without loss of efficiency. While this is perhaps best illustrated by the direct coupling experiments presented in this manuscript, it is also clear that there are strategies for TEV immobilization, such as HALO tag immobilization, to which the enzyme is highly amenable. Finally, the protocols described in this manuscript are a blueprint for the production and immobilization of enzymes onto SPIONs. Thus, we anticipate that numerous other enzymes, including other highly specific proteases and enzymes with applications in organocatalysis, will be amenable to the techniques described herein.

ACKNOWLEDGEMENTS We acknowledge the Michael Hooker proteomics facility at UNC - Chapel Hill for assistance with proteomics experiments. We also thank Mr. Tom Fink (ECU Biology Imaging Core Facility) for assistance with nanoparticle freeze drying protocol.

FUNDING ACS Petroleum Research Fund 57850-DNI4, ECU Department of Chemistry, Thomas Harriot College of Arts and Sciences, and the ECU Division of Research Development and Economic Engagement.

COMPETING INTERESTS STATEMENT The authors have no competing interests. 


\section{REFERENCES}

1. Kapust, R. B. and Waugh, D. S. "Controlled Intracellular Processing of Fusion Proteins by TEV Protease." Protein expression and purification 19, no. 2 (2000): 312 318. doi:10.1006/prep.2000.1251

2. Kapust, R. B., Tozser, J., Fox, J. D., Anderson, D. E., Cherry, S., Copeland, T. D., and Waugh, D. S. "Tobacco Etch Virus Protease: Mechanism of Autolysis and Rational Design of Stable Mutants with Wild-Type Catalytic Proficiency." Protein engineering 14, no. 12 (2001): 993-1000.

3. Berg, S. van den, Lofdahl, P.-A., Hard, T., and Berglund, H. "Improved Solubility of TEV Protease by Directed Evolution." Journal of biotechnology 121, no. 3 (2006): 291-298. doi:10.1016/j.jbiotec.2005.08.006

4. Fang, L., Jia, K.-Z., Tang, Y.-L., Ma, D.-Y., Yu, M., and Hua, Z.-C. "An Improved Strategy for High-Level Production of TEV Protease in Escherichia Coli and Its Purification and Characterization." Protein expression and purification 51, no. 1 (2007): 102-109. doi:10.1016/j.pep.2006.07.003

5. Wehr, M. C., Laage, R., Bolz, U., Fischer, T. M., Grunewald, S., Scheek, S., Bach, A., Nave, K.-A., and Rossner, M. J. "Monitoring Regulated Protein-Protein Interactions Using Split TEV." Nature methods 3, no. 12 (2006): 985-993. doi:10.1038/nmeth967

6. Wang, W., Wildes, C. P., Pattarabanjird, T., Sanchez, M. I., Glober, G. F., Matthews, G. A., Tye, K. M., and Ting, A. Y. "A Light- and Calcium-Gated Transcription Factor for Imaging and Manipulating Activated Neurons" Nature Biotechnology 35, no. 9 (2017): 864-871. doi:10.1038/nbt.3909, Available at http://www.nature.com/doifinder/10.1038/nbt.3909

7. Raran-Kurussi, S. and Waugh, D. S. "A Dual Protease Approach for Expression and Affinity Purification of Recombinant Proteins." Analytical biochemistry 504, (2016): 30-37. doi:10.1016/j.ab.2016.04.006

8. Miladi, B., Marjou, A. El, Boeuf, G., Bouallagui, H., Dufour, F., Martino, P. Di, and Elm'selmi, A. "Oriented Immobilization of the Tobacco Etch Virus Protease for the Cleavage of Fusion Proteins" Journal of Biotechnology 158, no. 3 (2012): 97-103. doi:10.1016/j.jbiotec.2012.01.010, Available at

http://dx.doi.org/10.1016/j.jbiotec.2012.01.010

9. Raynes, J. K., Domigan, L. J., Pearce, F. G., and Gerrard, J. A. "Immobilization of Tobacco Etch Virus (TEV) Protease on a High Surface Area Protein Nanofibril Scaffold." Biotechnology progress 34, no. 6 (2018): 1506-1512. doi:10.1002/btpr.2670

10. Dadfar, S. M., Roemhild, K., Drude, N. I., Stillfried, S. von, Knuchel, R., Kiessling, F., and Lammers, T. "Iron Oxide Nanoparticles: Diagnostic, Therapeutic and Theranostic Applications." Advanced drug delivery reviews 138, (2019): 302-325. doi:10.1016/j.addr.2019.01.005

11. Mok, H. and Zhang, M. "Superparamagnetic Iron Oxide Nanoparticle-Based Delivery Systems for Biotherapeutics." Expert opinion on drug delivery 10, no. 1 
12. Fu, C., Donovan, W. P., Shikapwashya-Hasser, O., Ye, X., and Cole, R. H. "Hot Fusion: An Efficient Method to Clone Multiple DNA Fragments as Well as Inverted Repeats without Ligase." PloS one 9, no. 12 (2014): e115318.

doi:10.1371/journal.pone.0115318

13. Chivers, C. E., Crozat, E., Chu, C., Moy, V. T., Sherratt, D. J., and Howarth, M. "A Streptavidin Variant with Slower Biotin Dissociation and Increased Mechanostability." Nature methods 7, no. 5 (2010): 391-393. doi:10.1038/nmeth.1450

14. Mruk, D. D. and Cheng, C. Y. "An Inexpensive Alternative to Commercially Available Kits Enhanced Chemiluminescence ( ECL) for Routine Immunoblotting" Spermatogenesis 1, no. 2 (2011): 121-122. doi:10.4161/spmg.1.2.16606

15. Tropea, J. E., Cherry, S., and Waugh, D. S. "Expression and Purification of Soluble His(6)-Tagged TEV Protease." Methods in molecular biology (Clifton, N.J.) 498, (2009): 297-307. doi:10.1007/978-1-59745-196-3_19

16. Dobber, J. and Pohl, M. "HaloTag: Evaluation of a Covalent One-Step Immobilization for Biocatalysis." Journal of biotechnology 241, (2017): 170-174. doi:10.1016/j.jbiotec.2016.12.004

17. Piran, U. and Riordan, W. J. "Dissociation Rate Constant of the BiotinStreptavidin Complex." Journal of immunological methods 133, no. 1 (1990): 141-143. doi:10.1016/0022-1759(90)90328-s

18. Olson, A. C. and Stanley, W. L. "Lactase and Other Enzymes Bound to a PhenolFormaldehyde Resin with Glutaraldehyde." Journal of agricultural and food chemistry 21, no. 3 (1973): 440-445. doi:10.1021/jf60187a001

19. Linko, Y. Y. and Pohjola, L. "A Simple Entrapment Method for Immobilizing Enzymes within Cellulose Fibers." FEBS letters 62, no. 1 (1976): 77-80. doi:10.1016/0014-5793(76)80020-8

20. Kim, Y.-Y., Bang, Y., Lee, A.-H., and Song, Y.-K. "Multivalent Traptavidin-DNA Conjugates for the Programmable Assembly of Nanostructures." ACS nano 13, no. 2 (2019): 1183-1194. doi:10.1021/acsnano.8b06170

21. Sorensen, H. P., Sperling-Petersen, H. U., and Mortensen, K. K. "A Favorable Solubility Partner for the Recombinant Expression of Streptavidin." Protein expression and purification 32, no. 2 (2003): 252-259. doi:10.1016/j.pep.2003.07.001

22. Motejadded, H., Kranz, B., Berensmeier, S., Franzreb, M., and Altenbuchner, J. "Expression, One-Step Purification, and Immobilization of HaloTag(TM) Fusion Proteins on Chloroalkane-Functionalized Magnetic Beads." Applied biochemistry and biotechnology 162, no. 7 (2010): 2098-2110. doi:10.1007/s12010-010-8985-1

23. Hu, Q., Katti, P. S., and Gu, Z. "Enzyme-Responsive Nanomaterials for Controlled Drug Delivery.” Nanoscale 6, no. 21 (2014): 12273-12286. doi:10.1039/c4nr04249b 
A

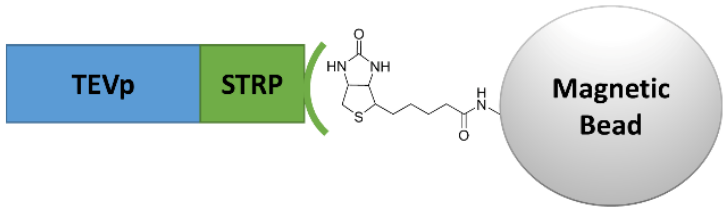

B

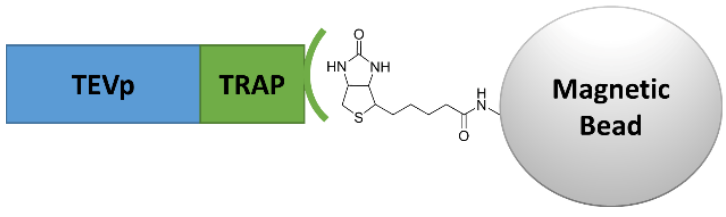

C

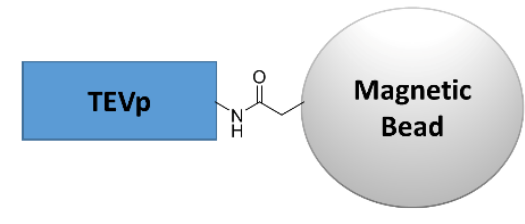

D

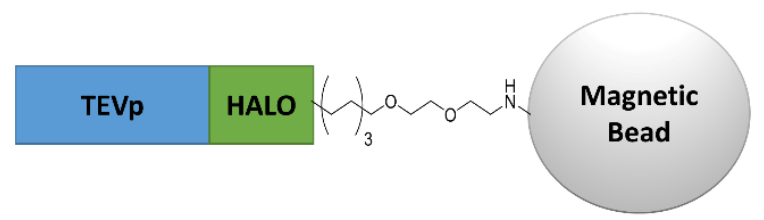

Figure 1. Tobacco Etch Virus (TEV) protease protein fusions and immobilization methods $A$. TEVstreptavidin (STRP) fusion - bead complex. B. TEV-TRPTavidin (TRAP) fusion - bead complex. C. Direct TEVp coupling via NHS-ester chemistry. D. TEV-HALO tag fusion - bead complex. 
A

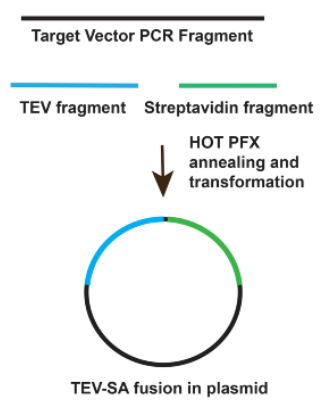

B

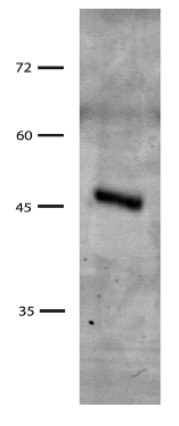

C

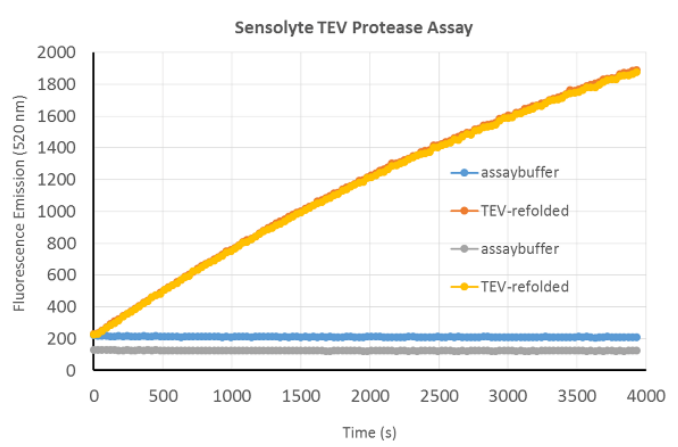

D
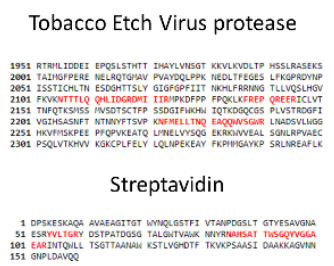

Figure 2. Cloning and characterization of a TEV-SA fusion protein. A. Cloning strategy for assembly of MPB-TEVp-STRPT ORF in the vector pNic28. B. SDS-PAGE of TEVpSTRPT fusion protein isolated from inclusion bodies and refolded. C. Activity of the refolded TEVp-STRPT fusion protein with the SensoLyte® TEV Protease assay. D. Peptide fragments (red highlights) identified with tryptic digest and MS analysis of the recovered TEVp-STRPT fusion protein. 


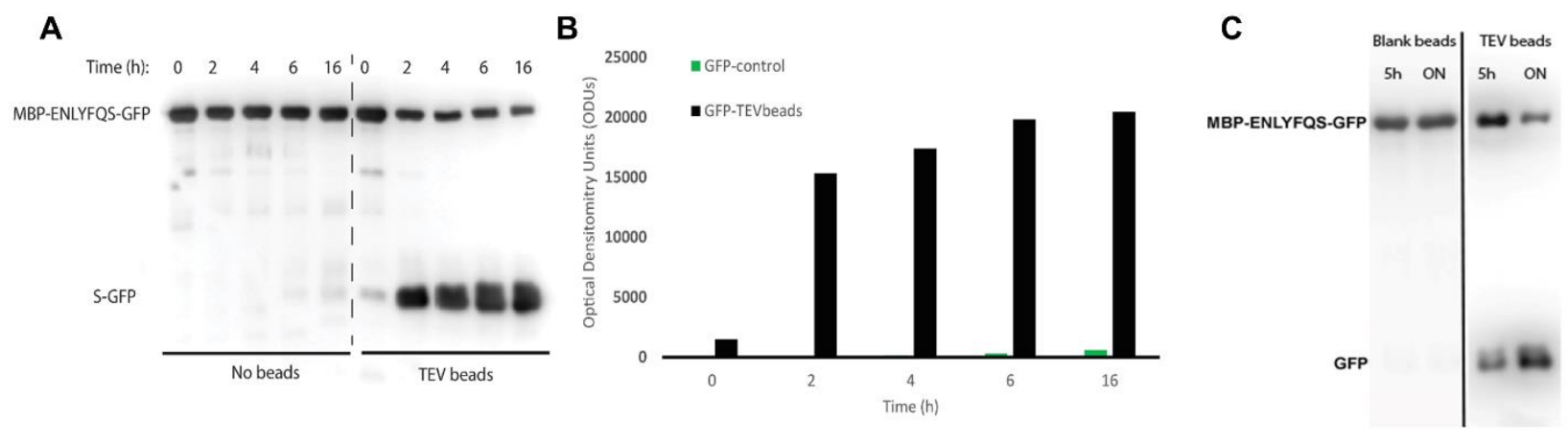

Figure 3. Timecourse of TEV bead activity. A. Western blot ( $\alpha-\mathrm{GFP})$ of TEV bead cleavage of a MBP-GFP protein fusion containing the ENLYFQS site $(50 \mu \mathrm{L}$ TEV SPIONs; $200 \mu \mathrm{L}$ of 0.1 $\mathrm{mg} / \mathrm{mL}$ protein substrate; $10 \mathrm{mM}$ DTT). Blank (control) beads do not induce substrate proteolysis, while TEV beads induce efficient substrate cleavage after overnight incubation at $30{ }^{\circ} \mathrm{C}$ (left panel). B. Densitometry results of appearance of S-GFP cleavage product compare TEV SPION induced cleavage (dark bars) with background, buffer only substrate cleavage (green bars). C. Western blot ( $\alpha$-GFP) of TEVp bead cleavage of a MBP-GFP protein fusion containing the ENLYFQS site. SPIONs loaded with a TEVp-free bacterial lysate (left blot) do not induce substrate proteolysis, while SPIONs incubated with a TEV-STRPT containing bacterial lysate (right blot) induce substrate cleavage. 
A

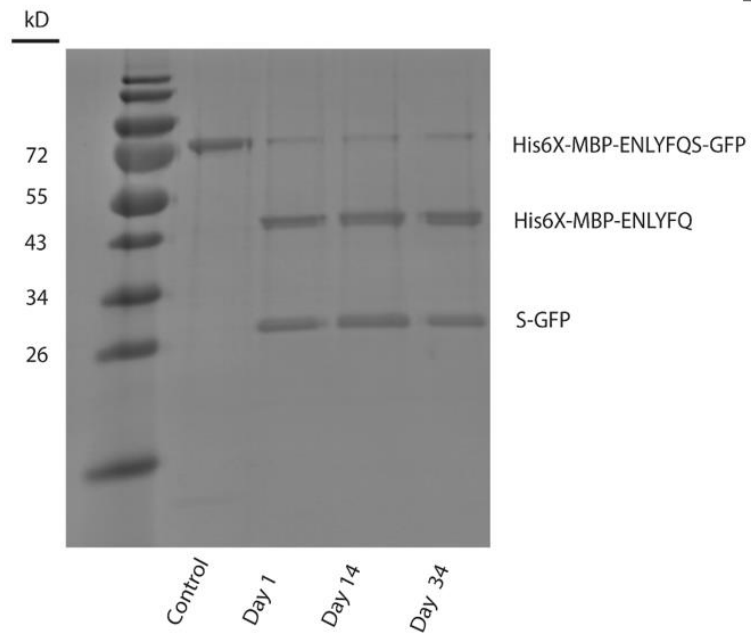

B

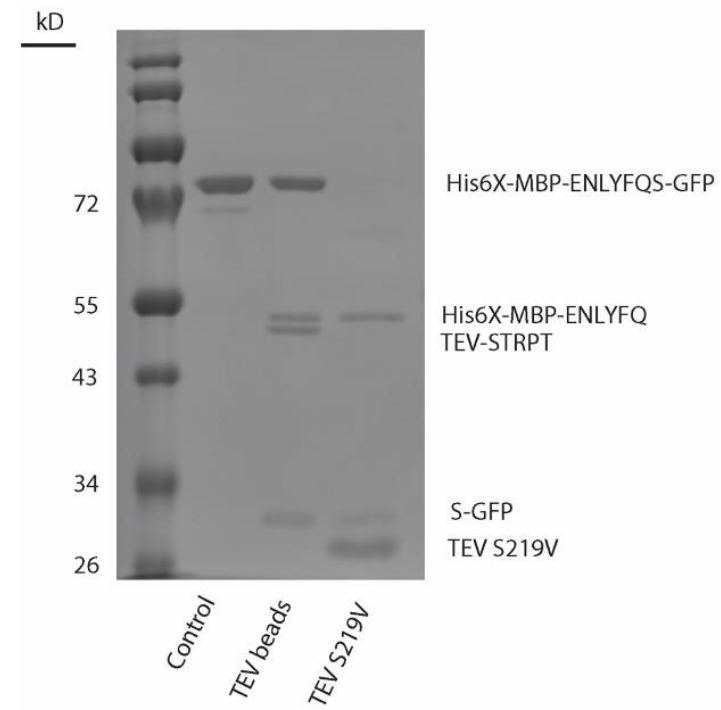

Figure 4 A. TEV bead longevity. TEV beads remain active for at least one month in cold storage $\left(4{ }^{\circ} \mathrm{C}\right.$; TEV storage buffer). Gradient SDS-PAGE gel shows test sample cleavage after overnight incubation. $200 \mu \mathrm{L}$ of a $74.6 \mathrm{kD}$ substrate protein $(0.1 \mathrm{mg} / \mathrm{mL})$ is efficiently cleaved $(90 \%+/-5)$ after overnight incubation with $50 \mu \mathrm{L}$ of TEV beads and $10 \mathrm{mM}$ DTT at $30{ }^{\circ} \mathrm{C}$. B. TEV bead protein fusion loss. TEV beads demonstrate protein loss that can be detected via SDS-PAGE. Two bands appear near $50 \mathrm{kD}$ in the TEV SPION sample, but only a single fragment appears near $50 \mathrm{kD}$ in the TEV S219V sample. In this assay, MBP-GFP substrate was incubated for $2 \mathrm{~h}$ with free TEV or with $50 \mu \mathrm{L}$ of day old TEV SPIONs for $2 \mathrm{~h}$ at $30{ }^{\circ} \mathrm{C}$. 


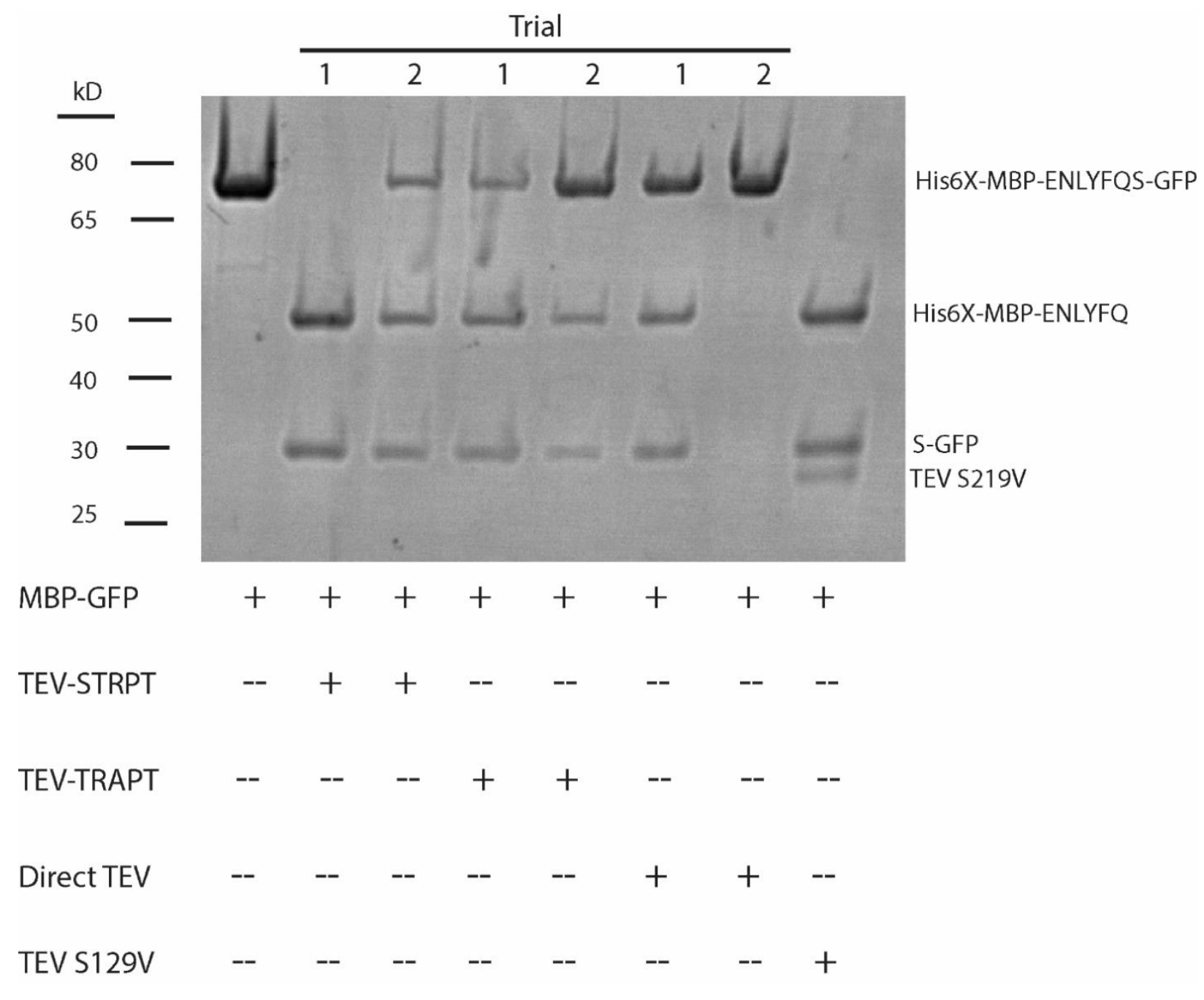

Figure 5 Performance of TEVp protein fusions and non-directionally immobilized TEVp over two successive assays $(20 \mu \mathrm{L}$ TEV SPIONs; $80 \mu \mathrm{L}$ of $0.2 \mathrm{mg} / \mathrm{mL}$ protein substrate; $5 \mathrm{mM}$ BME; Overnight incubation at $30 \mathrm{deg}$ C). Protease activity of TEV-STRPT (Lane 2-3) and TEV-TRPT (Lane 4-5) protein fusions on biotinylated SPIONs is compared to activity of TEV S219V directly coupled to NHS-functionalized SPIONs (Lane 6-7). Unbound TEVp S219V is used as a positive control (Lane 8). While both TEV-STRPT and TEV-TRPT SPIONs retain some activity over repeat usage (TEV-STRPT: trial 1: 100\%, trial 2: $41 \%(+/-5)$; TEV-TRPT: trial 1: $49 \%(+/-6)$, trial 2: $25 \%(+/-4)$, directly coupled TEV lost all protease activity after a single use. \% substrate cleavage by optical densitometry of His6X-MBP-ENLYFQ cleavage fragment from three replicate measurements (ImageJ). 
A

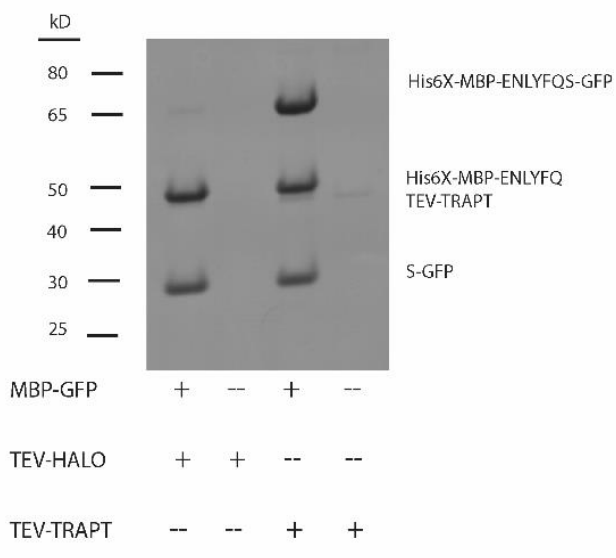

B

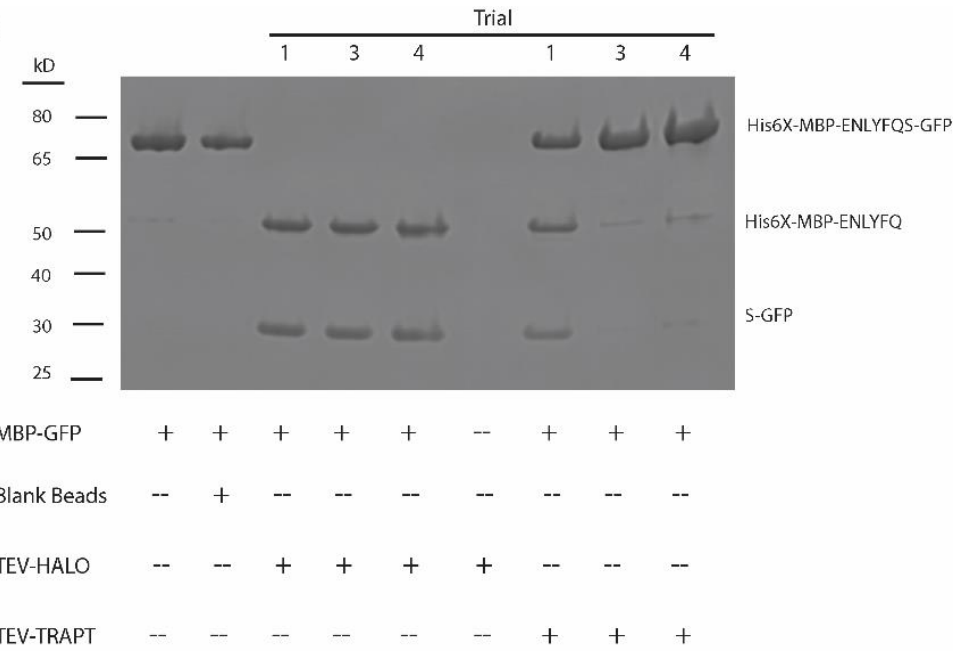

Figure 6 Performance of TEV-HALO protein fusions versus TEV-TRPT protein fusions. A. TEV-HALO SPIONs give near complete cleavage of the MBP-GFP substrate (lane 1), while TEV-TRPT SPIONs give only partial cleavage of the substrate (Lane 3). In addition, loss of TEV-TRPT from SPIONs is apparent (lane 4), whereas there is no observable protein fusion loss with the TEV-HALO SPIONs (lane 2). B. TEV-HALO spions demonstrate efficient cleavage over successive applications (Lanes 3 - 5; substrate only and blank bead + substrate controls are shown in Lanes 1 -2), whereas TEV-TRPT SPIONs lose rapidly lose activity with multiple applications (Lanes 7-9). All assays comprised of $20 \mu \mathrm{L}$ TEV SPIONs; $80 \mu \mathrm{L}$ of 0.2 $\mathrm{mg} / \mathrm{mL}$ protein substrate; $5 \mathrm{mM} \beta \mathrm{ME}$; with overnight incubation at $30{ }^{\circ} \mathrm{C}$. 


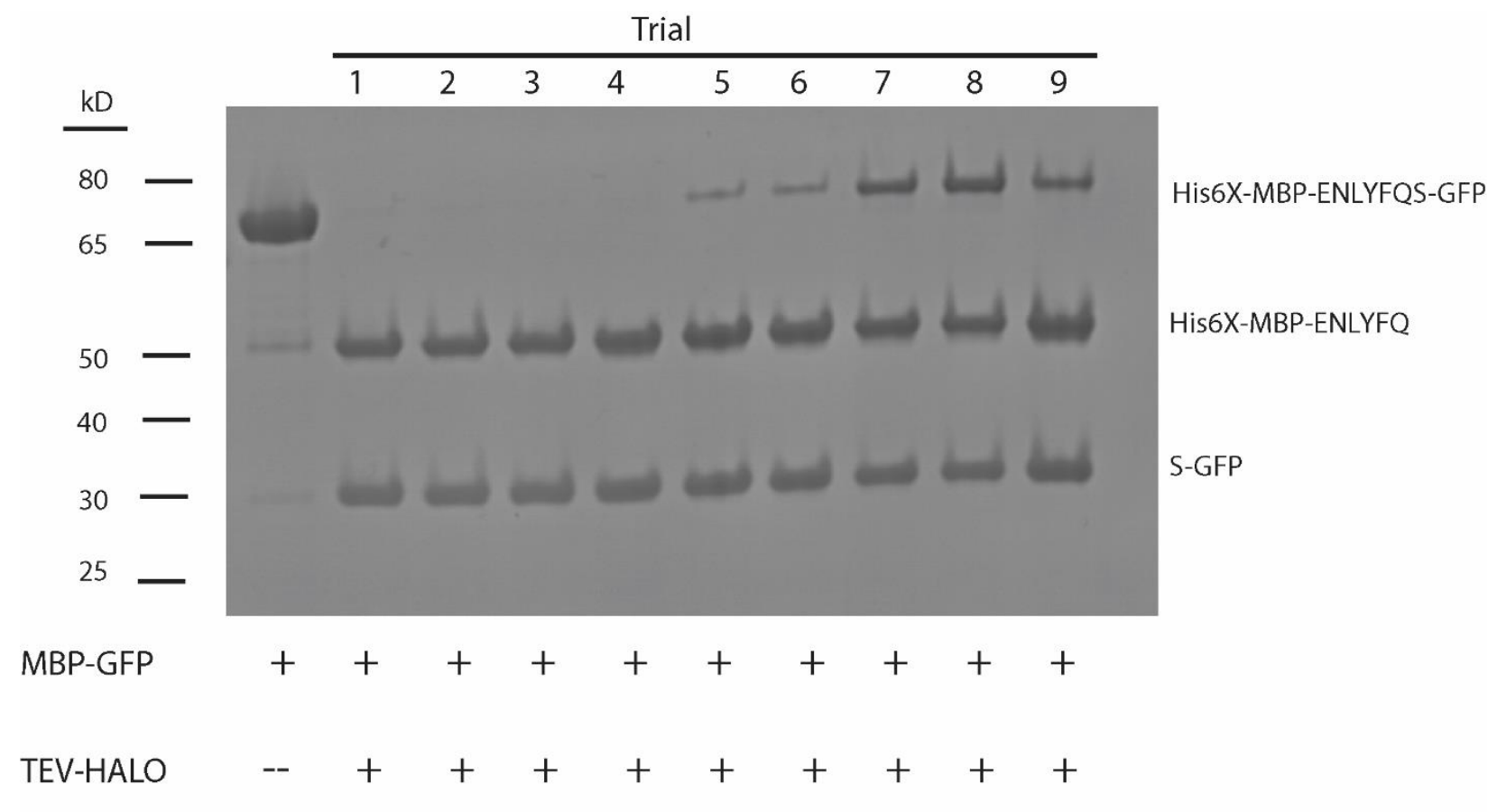

Figure 7 Repeat application of TEV-HALO protein fusion. Substrate only control (Lane 1) is compared to nine repeated applications of a $20 \mathrm{uL}$ aliquot of TEV-HALO beads (Lanes $2-$ 10). Only after six trials was a significant decrease in TEVp activity is observed $(92(+/-2) \%$ substrate cleaved), representing a significant improvement over the performance of the TEVSTRPT and TEV-TRPT SPIONs. Trials 7-9 had a further reduction in \% substrate cleaved $(68(+/-3) \%)$. All assays comprised of $20 \mu \mathrm{L}$ TEV SPIONs; $80 \mu \mathrm{L}$ of $0.2 \mathrm{mg} / \mathrm{mL}$ protein substrate; $5 \mathrm{mM}$ BME; with overnight incubation at $30{ }^{\circ} \mathrm{C}$. \% substrate cleavage determined from three replicate measurements by optical densitometry of intact MBP-GFP (ImageJ). 
Supporting Table 1. Peptides identified via Tryptic Digest and Proteomics Analysis of TEV-Streptavidin protein fusion

\section{TEV peptide identification}

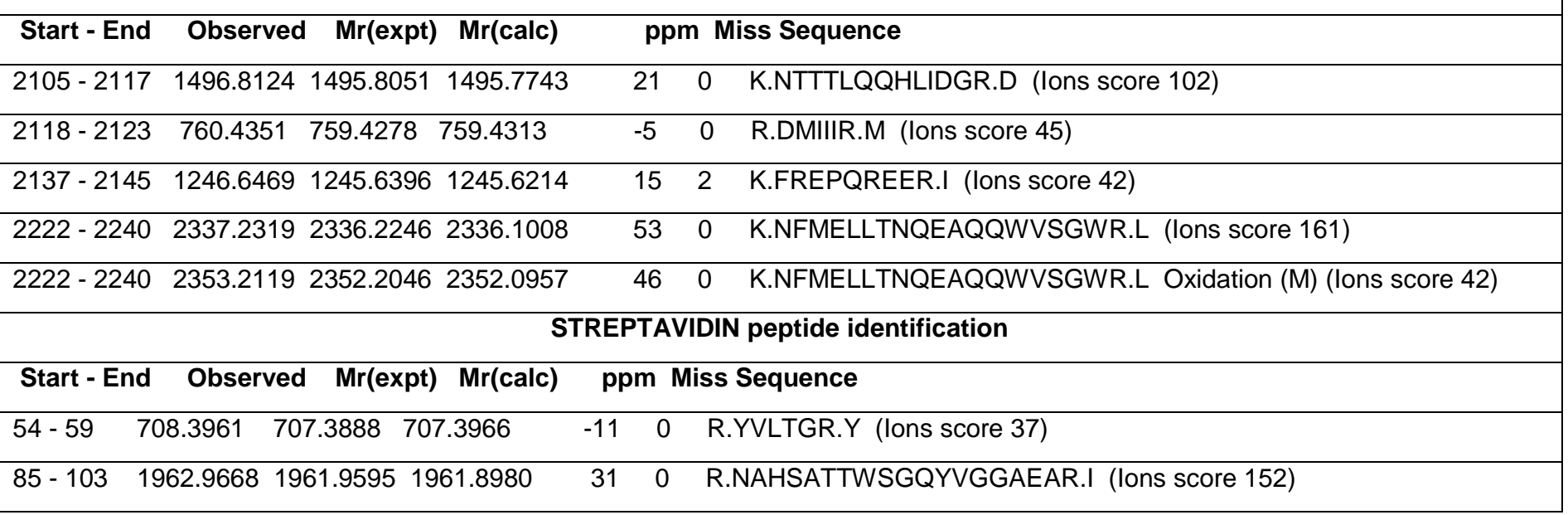




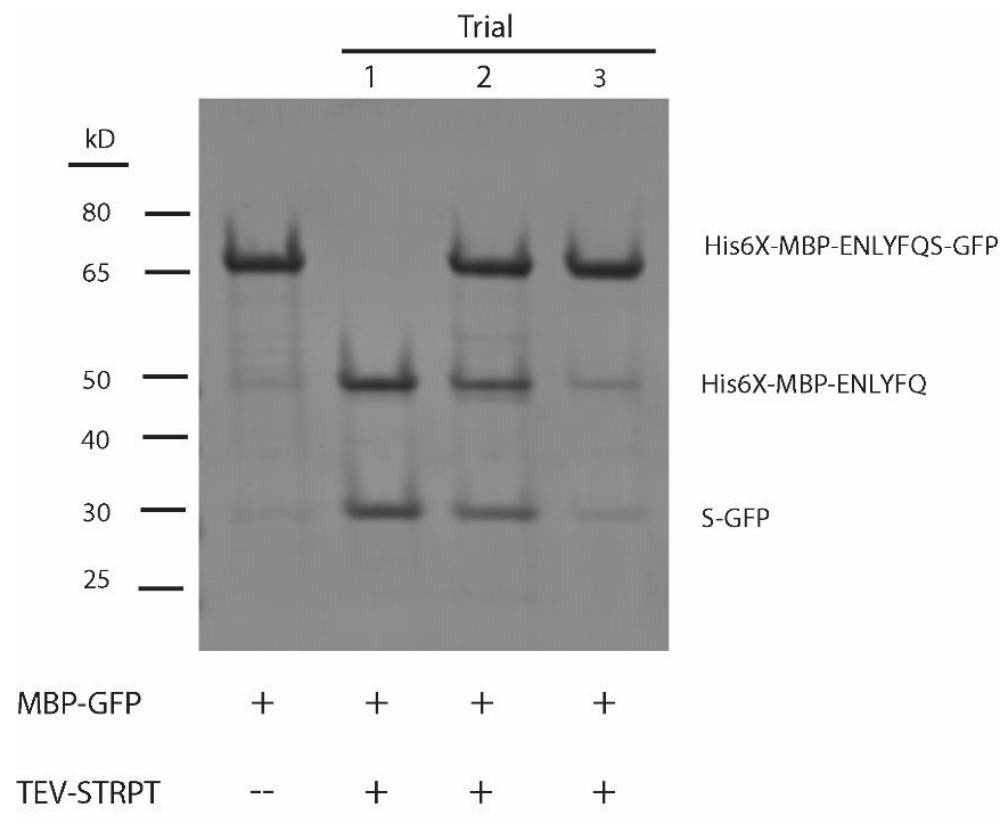

Supporting Figure 1. Repeat trials of TEV-STRPT beads. TEV-STRPT SPIONs were subjected to successive overnight MBP-GFP cleavage assays site (50 $\mu \mathrm{L}$ TEV SPIONs; $200 \mu \mathrm{L}$ of $0.1 \mathrm{mg} / \mathrm{mL}$ protein substrate; $10 \mathrm{mM}$ DTT). Significant loss of TEVp activity occurs over three successive trials (Trial 1: 100\%; Trial 2: 40(+/-5)\%; Trial 3: 15(+/$8) \%$, as determined by optical densitometry of His6X-MBP-ENLYFQ cleavage fragment from three replicate measurements (ImageJ). 
A

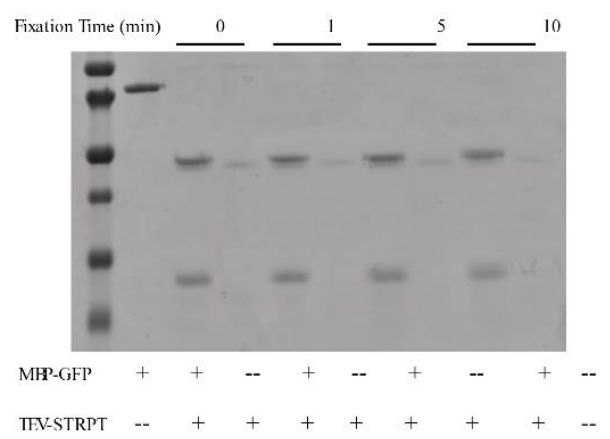

B

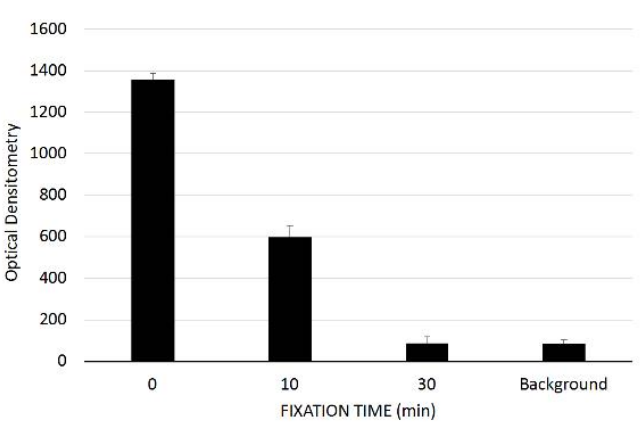

C

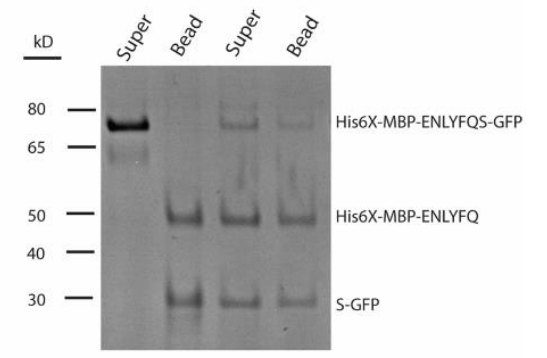

MBP-GFP

TEV-STRPT

TEV-STRPTFIX -- - + + +

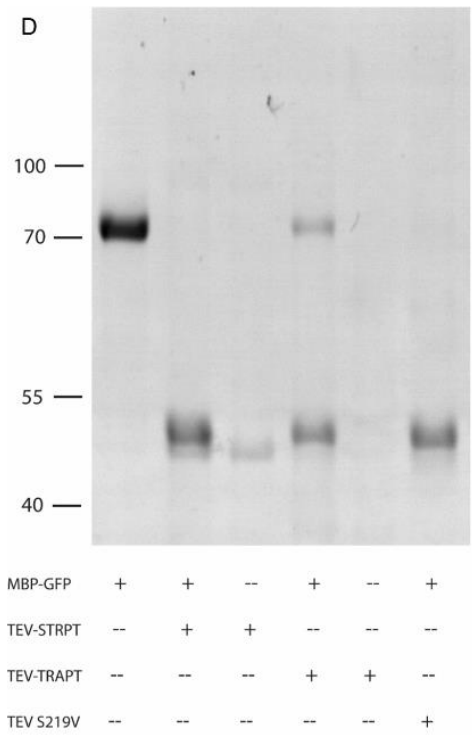

Supporting Figure 2. Impacts of PFA fixation on TEV activity and fusion protein loss. A. Assessement of TEV-STRPT SPIONs after fixation with $4 \%$ PFA for the indicated time intervals at 4 deg C. Fixed and unfixed beads were tested for activity (Lanes 2, 4, and 6) and fusion protein loss (Lanes 3, 5, and 7). Nonimmobilized TEV control shown in Lane 8. B. Optical densitometry of TEV-STRPT fusion protein loss. Error determined from three replicate measurements (ImageJ). C. Assessment of fusion protein activity associated with SPIONs (Lanes 2 and 4) and SPION storage buffer (Lanes 1 and 3) 10 days - post fixation. SPION storage buffer from PFA treated SPIONs had significant protease activity, indicating loss of fusion protein from SPIONs. D. TEV-STRPT and TEV-TRPT SPION activity (Lanes 2 and 4) and protein fusion loss (Lanes 2 and 5). Non-immobilized TEV control shown in Lane 6. TEV-TRPT SPIONs exhibit less protein fusion loss but have lower activity than TEV-STRPT SPIONs. All assays comprised of $50 \mu \mathrm{L}$ TEV SPIONs; $200 \mu \mathrm{L}$ of $0.1 \mathrm{mg} / \mathrm{mL}$ protein substrate; $10 \mathrm{mM}$ DTT; Overnight incubation at $30{ }^{\circ} \mathrm{C}$. 


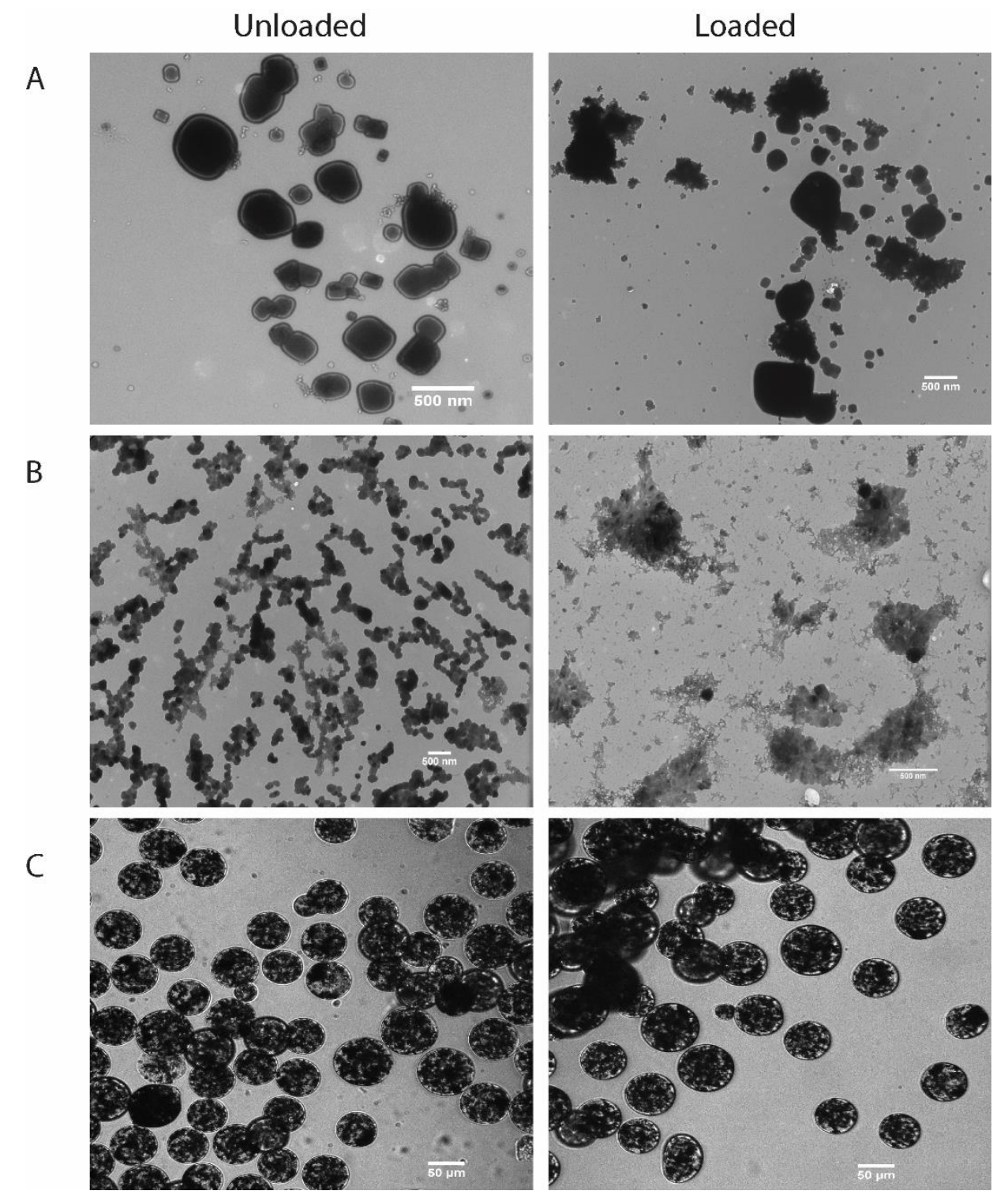

Supporting Figure 3. TEM and light microscopy images of SPIONs before and after protein immobilization. TEM images: A. Biotinlyated SPIONs before (left; mean diameter $=479.8(+/-103.3) \mathrm{nm})$ and after $($ right; mean diameter $=789.8(+/-151.3) \mathrm{nm})$ protein immobilization. B. NHS ester functionalized SPIONs before (left; mean diameter $=231.2(+/-58.8) \mathrm{nm})$ and after (right; mean diameter $=789.8(+/-151.3) \mathrm{nm})$ protein immobilization. Light microscope images: C. Macroporous cellulose encased SPIONs before (left; mean diameter $=63.0(+/-7.8)$ microns $)$ and after (right; mean diameter $=$ 63.4(+/- 8.9) microns) protein immobilization. 


\section{DNA sequences}

\section{MBP-TEVp-STRPT}

atgaaaactgaagaaggtaaactggtaatctggattaacggcgataaaggctataacggtctcgctgaagtcggtaaga aattcgagaaagataccggaattaaagtcaccgttgagcatccggataaactggaagagaaattcccacaggttgcggc aactggcgatggccctgacattatcttctgggcacacgaccgcttggtggctacgctcaatctggcctgttggctgaaatca ccccggacaaagcgttccaggacaagctgtatccgtttacctgggatgccgtacgttacaacggcaagctgattgcttacc cgatcgctgttgaagcgttatcgctgatttataacaaagatctgctgccgaacccgccaaaaacctgggaagagatcccg gcgctggataaagaactgaaagcgaaaggtaagagcgcgctgatgttcaacctgcaagaaccgtacttcacctggccg ctgattgctgctgacgggggttatgcgttcaagtatgaaaacggcaagtacgacattaaagacgtgggcgtggataacgct ggcgcgaaagcgggtctgaccttcctggttgacctgattaaaaacaaacacatgaatgcagacaccgattactccatcgc agaagctgcctttaataaaggcgaaacagcgatgaccatcaacggcccgtgggcatggtccaacatcgacaccagca aagtgaattatggtgtaacggtactgccgaccttcaagggtcaaccatccaaaccgttcgttggcgtgctgagcgcaggtat taacgccgccagtccgaacaaagagctggcaaaagagttcctcgaaaactatctgctgactgatgaaggtctggaagcg gttaataaagacaaaccgctgggtgccgtagcgctgaagtcttacgaggaagagttggcgaaagatccacgtattgccg ccaccatggaaaacgcccagaaaggtgaaatcatgccgaacatcccgcagatgtccgctttctggtatgccgtgcgtact gcggtgatcaacgccgccagcggtcgtcagactgtcgatgaagccctgaaagacgcgcagactaattcgagctcgaac aacaacaacaataacaataacaacaacctcgggatcgagggaaggggagaaaatctttatttcaaggtcatcatcatc atcatcatcatggagaaagcttgtttaaggggccgcgtgattacaacccgatatcgagcaccatttgtcatttgacgaatga atctgatgggcacacaacatcgttgtatggtattggatttggtcccttcatcattacaaacaagcacttgtttagaagaaataat ggaacactgttggtccaatcactacatggtgtattcaaggtcaagaacaccacgactttgcaacaacacctcattgatggg agggacatgataattattcgcatgcctaaggatttcccaccatttcctcaaaagctgaaatttagagagccacaaagggaa gagcgcatatgtcttgtgacaaccaacttccaaactaagagcatgtctagcatggtgtcagacactagttgcacattcccttc atctgatggcatattctggaagcattggattcaaaccaaggatgggcagtgtggcagtccattagtatcaactagagatgg gttcattgttggtatacactcagcatcgaatttcaccaacacaaacaattatttcacaagcgtgccgaaaaacttcatggaatt gttgacaaatcaggaggcgcagcagtgggttagtggttggcgattaaatgctgactcagtattgtgggggggccataaagt tttcatggtgaaacctgaagagcctttcagccagttaaggaagcgactcaactcatgaatcgtcgtcgccgtcgcatggat ccatccaaagattcaaaggcgcaggtatcagcagcagaagcgggtattactgggacatggtataaccagcttggttccac tttcatcgtcaccgcaggcgctgatggggctttaacaggtacttacgaaagtgcggtggggaacgcggaaagtcgctatgt attaactggacgctacgacagcgccccggccaccgacggtagtggaacggcactgggctggacggttgcttggaagaa caattatcgcaatgctcacagtgcgacgacatggtcagggcagtatgtggggggggccgaagcacgcatcaatacaca gtggttgttgacatccgggactaccgaggcgaacgcatggaaatcaacgctggtaggccatgatacatttacgaaggtaa aaccaagtgccgcgagtatcgacgcagctaagaaggctggtgtcaataatggcaatccgttagatgctgttcagcag 


\section{MBP-TEVp-TRPT}

atgaaaactgaagaaggtaaactggtaatctggattaacggcgataaaggctataacggtctcgctgaagtcggtaaga aattcgagaaagataccggaattaaagtcaccgttgagcatccggataaactggaagagaaattcccacaggttgcggc aactggcgatggccctgacattatcttctgggcacacgaccgctttggtggctacgctcaatctggcctgttggctgaaatca ccccggacaaagcgttccaggacaagctgtatccgtttacctgggatgccgtacgttacaacggcaagctgattgcttacc cgatcgctgttgaagcgttatcgctgatttataacaaagatctgctgccgaacccgccaaaaacctgggaagagatcccg gcgctggataaagaactgaaagcgaaaggtaagagcgcgctgatgttcaacctgcaagaaccgtacttcacctggccg ctgattgctgctgacgggggttatgcgttcaagtatgaaaacggcaagtacgacattaaagacgtgggcgtggataacgct ggcgcgaaagcgggtctgaccttcctggttgacctgattaaaaacaaacacatgaatgcagacaccgattactccatcgc agaagctgcctttaataaaggcgaaacagcgatgaccatcaacggcccgtgggcatggtccaacatcgacaccagca aagtgaattatggtgtaacggtactgccgaccttcaagggtcaaccatccaaaccgttcgttggcgtgctgagcgcaggtat taacgccgccagtccgaacaaagagctggcaaaagagttcctcgaaaactatctgctgactgatgaaggtctggaagcg gttaataaagacaaaccgctgggtgccgtagcgctgaagtcttacgaggaagagttggcgaaagatccacgtattgccg ccaccatggaaaacgcccagaaaggtgaaatcatgccgaacatcccgcagatgtccgcttctggtatgccgtgcgtact gcggtgatcaacgccgccagcggtcgtcagactgtcgatgaagccctgaaagacgcgcagactaattcgagctcgaac aacaacaacaataacaataacaacaacctcgggatcgagggaaggggagaaaatctttatttcaaggtcatcatcatc atcatcatcatggagaaagcttgtttaaggggccgcgtgattacaacccgatatcgagcaccatttgtcatttgacgaatga atctgatgggcacacaacatcgttgtatggtattggatttggtcccttcatcattacaaacaagcacttgtttagaagaaataat ggaacactgttggtccaatcactacatggtgtattcaaggtcaagaacaccacgacttgcaacaacacctcattgatggg agggacatgataattattcgcatgcctaaggatttcccaccatttcctcaaaagctgaaatttagagagccacaaagggaa gagcgcatatgtcttgtgacaaccaacttccaaactaagagcatgtctagcatggtgtcagacactagttgcacattcccttc atctgatggcatattctggaagcattggattcaaaccaaggatgggcagtgtggcagtccattagtatcaactagagatgg gttcattgttggtatacactcagcatcgaatttcaccaacacaaacaattatttcacaagcgtgccgaaaaacttcatggaatt gttgacaaatcaggaggcgcagcagtgggttagtggttggcgattaaatgctgactcagtattgtgggggggccataaagt tttcatggtgaaacctgaagagccttttcagccagttaaggaagcgactcaactcatgaatcgtcgtcgccgtcgccgtcgt cgccgtcgccgtatggctgaagctggtatcaccggcacctggtacaaccagctgggatccaccttcatcgttaccgctggt gctgacggtgctctgaccggtacctacgaatccgctgttggtaacgctgaaGGCGATtacgttctgaccggtcgttacg actccgctccggctaccgacggttccggaaccgctctgggttggaccgttgcttggaaaaacaactaccgtaacgctcact ccgctaccacctggtctggccagtacgttggtggtgctgaagctcgtatcaacacccagtggttgttgacctccggcaccac cgaagctaacgcgtggaaatccaccctggttggtcacgacaccttcaccaaagttaaaccgtccgctgcttcccatcacca tcaccaccattaataa 


\section{MBP-TEV-HALO}

atgaaaactgaagaaggtaaactggtaatctggattaacggcgataaaggctataacggtctcgctgaagtcggtaaga aattcgagaaagataccggaattaaagtcaccgttgagcatccggataaactggaagagaaattcccacaggttgcggc aactggcgatggccctgacattatcttctgggcacacgaccgctttggtggctacgctcaatctggcctgttggctgaaatca ccccggacaaagcgttccaggacaagctgtatccgtttacctgggatgccgtacgttacaacggcaagctgattgcttacc cgatcgctgttgaagcgttatcgctgatttataacaaagatctgctgccgaacccgccaaaaacctgggaagagatcccg gcgctggataaagaactgaaagcgaaaggtaagagcgcgctgatgttcaacctgcaagaaccgtacttcacctggccg ctgattgctgctgacgggggttatgcgttcaagtatgaaaacggcaagtacgacattaaagacgtgggcgtggataacgct ggcgcgaaagcgggtctgaccttcctggttgacctgattaaaaacaaacacatgaatgcagacaccgattactccatcgc agaagctgcctttaataaaggcgaaacagcgatgaccatcaacggcccgtgggcatggtccaacatcgacaccagca aagtgaattatggtgtaacggtactgccgaccttcaagggtcaaccatccaaaccgttcgttggcgtgctgagcgcaggtat taacgccgccagtccgaacaaagagctggcaaaagagttcctcgaaaactatctgctgactgatgaaggtctggaagcg gttaataaagacaaaccgctgggtgccgtagcgctgaagtcttacgaggaagagttggcgaaagatccacgtattgccg ccaccatggaaaacgcccagaaaggtgaaatcatgccgaacatcccgcagatgtccgcttctggtatgccgtgcgtact gcggtgatcaacgccgccagcggtcgtcagactgtcgatgaagccctgaaagacgcgcagactaattcgagctcgaac aacaacaacaataacaataacaacaacctcgggatcgagggaaggggagaaaatctttatttcaaggtcatcatcatc atcatcatcatggagaaagcttgtttaaggggccgcgtgattacaacccgatatcgagcaccatttgtcatttgacgaatga atctgatgggcacacaacatcgttgtatggtattggatttggtcccttcatcattacaaacaagcacttgtttagaagaaataat ggaacactgttggtccaatcactacatggtgtattcaaggtcaagaacaccacgactttgcaacaacacctcattgatggg agggacatgataattattcgcatgcctaaggatttcccaccatttcctcaaaagctgaaatttagagagccacaaagggaa gagcgcatatgtcttgtgacaaccaacttccaaactaagagcatgtctagcatggtgtcagacactagttgcacattcccttc atctgatggcatattctggaagcattggattcaaaccaaggatgggcagtgtggcagtccattagtatcaactagagatgg gttcattgttggtatacactcagcatcgaatttcaccaacacaaacaattatttcacaagcgtgccgaaaaacttcatggaatt gttgacaaatcaggaggcgcagcagtgggttagtggttggcgattaaatgctgactcagtattgtgggggggccataaagt tttcatggtgaaacctgaagagcctttcagccagttaaggaagcgactcaactcatgaatcgtcgtcgccgtcgcatggca gaaatcggtactggctttccattcgacccccattatgtggaagtcctgggcgagcgcatgcactacgtcgatgttggtccgc gcgatggcacccctgtgctgttcctgcacggtaacccgacctcctcctacgtgtggcgcaacatcatcccgcatgttgcacc gacccatcgctgcattgctccagacctgatcggtatgggcaaatccgacaaaccagacctgggttatttcttcgacgacca cgtccgcttcatggatgccttcatcgaagccctgggtctggaagaggtcgtcctggtcattcacgactggggctccgctctg gtttccactgggccaagcgcaatccagagcgcgtcaaaggtattgcatttatggagttcatccgccctatcccgacctggga cgaatggccagaatttgcccgcgagaccttccaggccttccgcaccaccgacgtcggccgcaagctgatcatcgatcag aacgttttatcgagggtacgctgccgatgggtgtcgtccgcccgctgactgaagtcgagatggaccattaccgcgagccg ttcctgaatcctgttgaccgcgagccactgtggcgcttcccaaacgagctgccaatcgccggtgagccagcgaacatcgt cgcgctggtcgaagaatacatggactggctgcaccagtcccctgtcccgaagctgctgttctggggcaccccaggcgttct gatcccaccggccgaagccgctcgcctggccaaaagcctgcctaactgcaaggctgtggacatcggcccgggtctgaa tctgctgcaagaagacaacccggacctgatcggcagcgagatcgcgcgctggctgtcgacgctcgagatttccggc 